\title{
OPTIMIZATION OF ADAPTIVE MODULATION AND CODING FOR VIDEO TRANSMISSION OVER A SINGLE-HOP WIRELESS NETWORK
}

\author{
Vijay C $\mathbf{P}^{1}$, Supriya ${ }^{2}$, Ankitha $\mathrm{K}^{3}$, Renita Blossom Monteiro ${ }^{4}$ \\ ${ }^{I} P$ G Scholar, Department of CSE, SCEM Mangalore, Karnataka, INDIA \\ ${ }^{2}$ Assistant Professor Department of CSE PCET Belthangady, Karnataka, INDIA \\ ${ }^{3}$ Assistant Professor Department of CSE SCEM, Mangalore, Karnataka, INDIA \\ ${ }^{4}$ Assistant Professor Department of CSE PCET, Belthangady, Karnataka, INDIA
}

\begin{abstract}
Adaptive modulation and coding (AMC) is a method in which automatic change in the modulation and forward error correction of a link to compensate for changes in link conditions due to the channel fading or interference. When compared with links using fixed coding, AMC can increase the throughput of a robust link by allowing it to dynamically adjust to a less robust modulation/coding resulting in higher throughput. In this project work, AMC is being considered for video transmission over wireless networks using NS2. Evalvid tool is used on NS2 for video transmission. 16-QAM, 64-QAM, BPSK and QPSK modulations are used and adapted in this simulation and analysis. Simulations are conducted for single-hop networks by considering sink node movement. In this project, the fixed modulation and AMC is being compared. From the simulation, it is observed that AMC yields better throughput, packet delivery ratio and PSNR compared to the fixed modulation.
\end{abstract}

Keywords-16-QAM; 64-QAM; AMC; BPSK; Evalvid; QPSK;

\section{INTRODUCTION}

Multimedia communications has significantly facilitated and enriched people's daily life. Because of the success of both wireless networks and video compression technology, multimedia applications especially real-time video communications is the main theme of the next-generation wireless Internet. The difference between wired and wireless networks is the cause of packet losses and mobility factor. In wired networks packet losses occurs because of congestion in the path between the source and the destination, packet losses in wireless networks occur mainly due to multipath fading, and the interference from the neighbouring transmissions. Mobility in wireless networks includes a number of additional barriers in multimedia data transmission

\subsection{Challenges in Multimedia Communication}

Bringing delay-sensitive and loss-tolerant for multimedia applications over wireless networks is highly challenging task because of,

- High packet loss and bit error rate .

- Limitation in bandwidth and fluctuations in available bandwidth, packet loss rate, delay and jitter.

- The mobile devices are power constrained.

\subsection{Definition of Modulation}

The process that make changes in one or more parameters of base communications signal such as amplitude, frequency, phase or angle of the signal, for the purpose of encoding information into the signal that is suitable for transmission.

\subsection{Adaptive Modulation and Coding}

"AMC is a technology which can automatically change the modulation and forward error correction of a link to compensate for changes in link conditions due to the channel fading or interference". When compared with links designed using fixed coding, AMC can increase the throughput of a robust link by allowing it to dynamically adjust to a less robust modulation/coding resulting in higher throughput. The different variants of modulation are used in various communication scenarios, in order to meet specific data rate performance as shown in Figure 1.1. However, while using modulation technique 64-QAM requires better signal-to-noise ratios in order to overcome from any interference and maintain a certain BER.

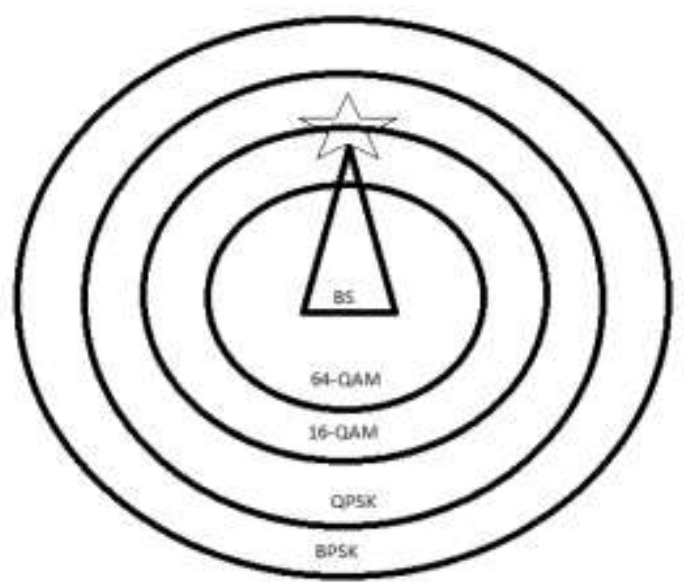

Fig -1.1 Modulation order for different users of Base Station 


\section{LITERATURE SURVEY}

\subsection{AMC Approaches}

In the survey paper [1], the performance of cross-layer optimization solutions for real-time video communications over three types of wireless networks, i.e., single-hop wireless networks, multi-hop wireless networks, and cooperative wireless networks are investigated. The joint optimization of the video coding parameters of an H.264 codec at the application layer and the transmission parameters at lower layers in a cross-layer manner are considered. From the point of view of end users, the end-toend video quality is the objective function for any optimization framework for video streaming. Hence the "recursive optimal per-pixel estimate" (ROPE) method in the H.264 video codec to calculate the expected received video distortion under the video transmission delay constraints.

In [2][3], a joint adaptive modulation and coding at the physical layer, and an ARQ at the data link layer considered for a single hop networks to maximize spectral efficiency under delay and error performance constraints. In [4], AMC techniques used in WiMAX based wireless networks to improve the network performance in case of non line-ofsight communications in urban environments. In [5], AMC scheme is used for IEEE 802.11n MIMO OFDM systems with convolution coding.. In this project AODV protocol is used and is explained in [8].

\subsection{Modulation Techniques Considered}

In this paper ,mainly concentrating on BPSK ,QPSK,16QAM and 64-QAM modulations [9][10]over wireless network.

\subsubsection{Binary Phase Shift Keying (BPSK)}

The simplest form of PSK is BPSK. There are two phases 0 and $\pi$ which are separateda by $180^{\circ}$ and can also known as 2-PSK. It is only able to modulate at $1 \mathrm{bit} / \mathrm{symbol}$ and so it is unsuitable for high data-rate applications. It is equivalent to 2-QAM modulation.

The general form of transmitted BPSK signal is as given below

$S_{B P S K}(t)=\sqrt{\frac{2 E_{b}}{T_{b}}} \cos \left[2 \pi f_{c} t+(1-n)\right] \quad n=$

$0,1 \quad 0 \leq \quad t \leq T_{b}$

where $E_{b}$ is the Energy per bit, $T_{b}$ is the bit duration and $f_{c}$ is the carrier frequency.

The probability of BER of BPSK can be calculated as

$P_{b}=Q\left(\sqrt{\frac{2 E_{b}}{N_{0}}}\right)$

It is also symbol error rate because one bit per symbol.

\subsubsection{Quadrature Phase Shift Keying (QPSK)}

QPSK [11] is known as quadriphase-PSK, 4-PSK, or 4QAM. With four phases, it can encode two bits per symbol and at the same BER, it transmits twice the data rate in a given bandwidth as compared to BPSK. The general form of QPSK Signal is as follows:

$$
\begin{aligned}
& S_{Q P S K}(t)=\sqrt{\frac{2 E_{s}}{T_{S}}} \cos \left[2 \pi f_{c} t+(n-1) \frac{\pi}{2}\right] \\
& 1,2,3,4 \quad 0 \leq t \leq T S
\end{aligned}
$$$$
n=
$$

where $T_{s}$ is the symbol duration.

QPSK has in-phase component of the signal uses the even (or odd) bits for modulating signal and quadrature component of the signal uses odd (or even) bits for modulating signal. BPSK is used on both carriers and so they can be independently demodulated. Hence, the probability of BER for QPSK is the same as for BPSK.

\subsubsection{Quadrature Amplitude Modulation(QAM)}

QAM [12] is a method which combines two amplitudemodulated signals to make a single channel, thereby doubling the effective bandwidth.

As a result, higher order versions of QAM are only used when there is a sufficiently high signal to noise ratio. In this paper 16-QAM and 64-QAM is used. Table1.gives a summary of the bit rates of QAM and PSK

Table 2.1 Bit rates for different forms of QAM and PSK

\begin{tabular}{|l|l|l|}
\hline Modulation & Bits per symbol & Symbol Rate \\
\hline BPSK & 1 & 1 bit rate \\
\hline QPSK & 2 & $1 / 2$ bit rate \\
\hline 16QAM & 4 & $1 / 4$ bit rate \\
\hline 64QAM & 6 & $1 / 6$ bit rate \\
\hline
\end{tabular}

\subsection{Evalvid Tool used for Video Transmission}

"EvalVid is a frameworka and tool-set for evaluation of the quality of video transmitted over a real or simulated communication networks[13]".It measures the QoS parameters of simulation networks like delay, jitter and loss rates and video quality by using PSNR metric. It supports MPEG-4,H.263 and H.264 codecs and also supports AAC makes use of PESQ or PEAQ for perceptual quality evaluation.

\subsection{Real Time Transport Protocol}

The Real-time Transport Protocol (RTP) [14][15] defines a standardized packet format for delivering audio and video over IP networks. It is designed for end-to-end, real-time, transfer of stream data.

\subsection{Video Coding}

There are three basic types of compressed video frames Explained in[16]:i.e I-Frame,P-Frame,B-Frame 


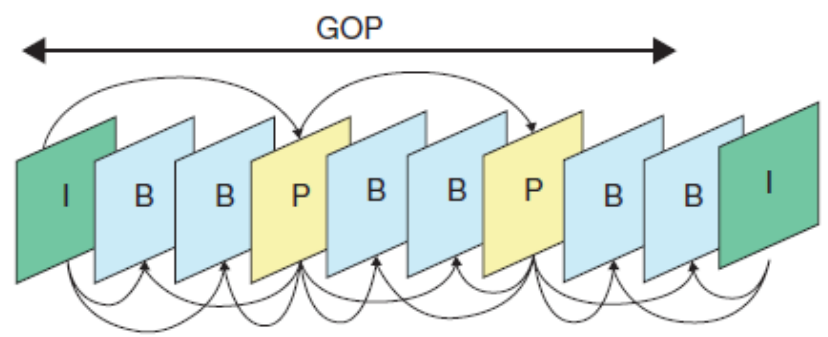

Fig -2.1 MPEG coding with G(9,3)

Generally, the entire video sequence can be decomposed into smaller units that are then coded together in the group of picture (GOP).A GOP pattern is characterized by two parameters, $\mathrm{G}(\mathrm{N}, \mathrm{M})$ in which $\mathrm{N}$ is the I-to-I frame distance, and $\mathrm{M}$ is the I-to-P frame distance. MPEG coding with $\mathrm{G}(9$, 3 ) as shown in figure 2.1 means that the GOP includes one I-aframe, two Paframes, and six Baframes. The second Iframe shown in Fig.2.1 indicates the beginning of the next GOP. The arrows indicate that the decoded B-frames and Pframes are dependent on the preceding or succeeding I- or P-frames.

\section{SYSTEM DESIGN}

The system model for video transmission over wireless networks in NS2 using Evalvid as shown in figure 31.

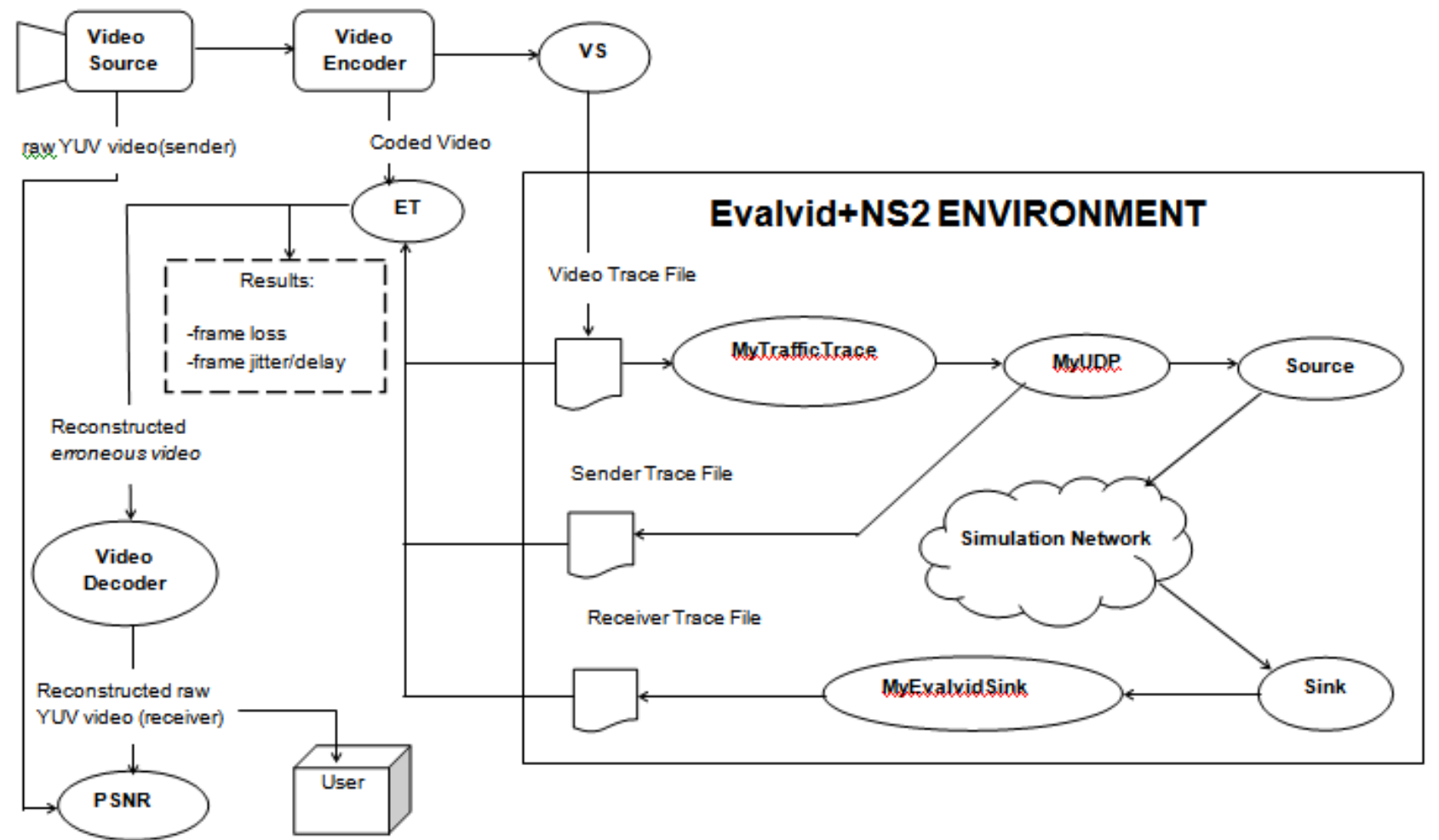

Fig -3.1 System model for video transmission over wireless networks in NS2 using Evalvid

The main components of Evalvid are as follows:

- Video Source : The file format available in online can be either in CIF with video resolution $352 \times 288$ or QCIF with video resolution 176 x 144 .

- VideoaEncoder and VideoaDecoder : Evalvid currently supports H.264, MPEG-4 and H.263 codecs. But ffmpeg and H.263 codecs does not support B-frames. Video Encoder encodes the file into a compressedavideo file and Video Decoder decodes the received vide frames into YUV format.

- Video Sender(VS) : Video Sender reads the compressed video file from the output of Video Encoder. Before transmitting video frames through UDP packets over wireless network, the Video sender fragments each video frame into smaller segments if the video frame size is larger than the Maximum Transmission Unit(MTU). It generates a video trace file containing information about every frame in the real video file.

- Evaluate Trace(ET) : Evaluate Trace is responsible for the task of evaluation. It generates a report of video frame/packet delay, frame/packet loss and frame/packet jitter by comparing the trace files including receiver trace file, sender trace file, video trace file and original encoded video file.

- $\quad$ Peak Signal to Noise Ratio (PSNR): PSNR is a metric to check the video quality. 
The connecting interfaces between NS-2 and Evalvid

- MyTrafficTrace : MyTrafficTrace reads the video trace file and extracts the video frame type and video frame size. Then it fragments the video frames into smaller video packets and sends these packets to the lower layers at appropriate time.

- MyUDP : MyUDP enhances the original UDP component in NS-2. It works as a tcpdump in a real network environment and generates sender trace file. It records the information of transmitted video packets i.e.,Timestamp, Packet ID, Packet Type and Payload Size of the packet.

- MyUDPSink : MyUDPSink is a receiving agent for video packets sent by MyUDP. It also generates the receiver trace file and records the information of received video packets

\subsection{Network Simulator Used}

Network simulator(NS)-2.35 is used. NS-2 is a discrete event driven network simulator mainly used for networking research. This tool is used to simulate the behaviour of wired and wireless networks. Table 3.1 shows the TCP/IP model used in this project.

Table 4.1 TCP/IP model

\begin{tabular}{||l|l||}
\hline \hline Application0Layer & Video Traffic(RTP) \\
\hline \hline Transport0Layer & UDP \\
\hline \hline Network0Layer & AODV protocol \\
\hline \hline Data Link0Layer & Simplex mode \\
\hline \hline Physical0Layer & AMC \\
\hline
\end{tabular}

\subsection{EvalVid - A Video Quality Evaluation Tool-Set}

EvalVid-2.7 version for linux contains binary files eg, etmp4, hist, miv, mos, mp4trace, psnr, vsgen. It also require ffmpeg and MP4Box to be downloaded separately. The whole package can be used to evaluate media over a simulated network using NS-2. To run it successfully ffmpeg, mp4box, mp4trace, etmp4 and psnr is required.

- ffmpeg: ffmpeg is a very fast video and audio converter that can also grab from a live audio/video source. Used to convert the yuv format to a .m4v format.

- mp4box: MP4Box is a multi-purpose command line tool to create and edit MPEG-4 Systems presentations and manipulate ISO-media files (MP4, 3GP, MOV) converts .m4v to a .mp4 file.

- mp4trace: mp4trace generates the trace file which is got using the details like packet mode or frame mode or protocol type, delay, etc. This trace file is sent over the simulated network.

- $\quad$ etmp4: This is used to generate the received video file which would be probably corrupted where all the frames that were corrupted or lost would be deleted.

- psnr: This calculates the peak signal to noise ratio between the two files (sent and received).

\section{IMPLEMENTATION}

\subsection{Installation of evalvid on ns-2}

Install the following packages before installing evalvid

- Download and install ffmpeg codec and x264 codec for linux

- Install libavcodec package is necessary for the linux platform to play audio and video file.

- Download and install GPAC library Copy include/gpac subdirectory to /usr/include. This library is used by etmp4.

- Install NS-2.34 or NS-2.35 package in linux platform and only these two packages have WirelessPhyExt and MAC 802.11Ext files.

- Download EvalVid package for NS-2 make the necessary changes as defined in README file

- Download latest Evalvid- 2.7 version for the linux platform contains necessary binaries for video transmission.

\subsection{Scenario of the Project}

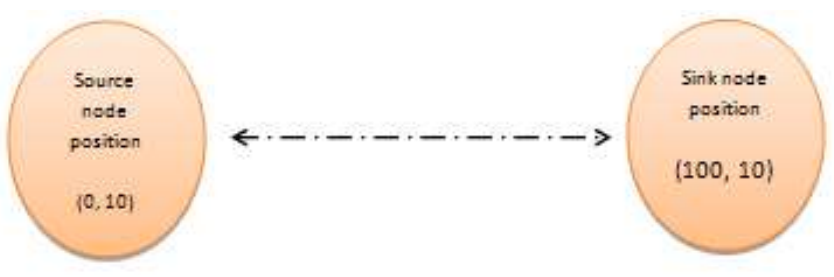

Fig 4.1 Network Scenario

Simulations are conducted in the NS-2.35 over single-hop network by considering the Sink node movement at a different speed and simulation time. In NS-2.35, the configuration specified for two nodes at $100 \mathrm{~m}$ distance in flat space with a size of $10000 \times 10000 \mathrm{~m}$ as shown in figure 3.2. At time 0.5 Seconds sink node starts moving at a given particular speed. Simulations are conducted for different sink node speed $10 \mathrm{~m} / \mathrm{s}, 15 \mathrm{~m} / \mathrm{s}, 20 \mathrm{~m} / \mathrm{s}, 25 \mathrm{~m} / \mathrm{s}$ and $30 \mathrm{~m} / \mathrm{s}$ and for each speed simulation time varied from 10 to 100 seconds. For each node speed and simulation time compared the performance of each modulation. As node move away from source node, at some particular simulation time the packets are gets dropping for each modulation. Note the simulation time when packets starts dropping. By using this simulation time it is easy to find the transmission range of each modulation. Therefore it is possible to adapt the different modulation based on transmission range. Different modulations are defined in WirelessPhy-Extension header as shown in table 4.1 being compared and adapted at physical layer. Routing protocol AODV is being considered 16QAM,64-QAM,BPSK and QPSK modulations are used in this project. Table 4.2 defines the configuration for wireless network. 
Table 4.1 Modulation parameters defined in WirelessPhyExt Header in NS-2.35

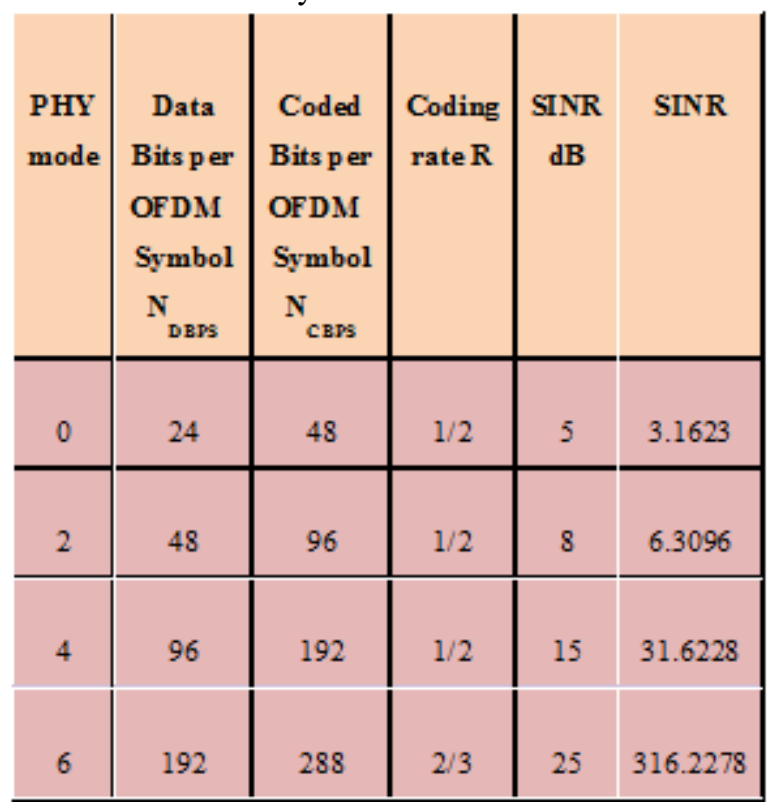

Table -4.2 Wireless Network Configuration

\begin{tabular}{|l|l||}
\hline Channel Type & Channel/WirelessChannel \\
\hline \hline Propagation Model & Propagation/TwoRayGround \\
\hline \hline $\begin{array}{l}\text { Network Interface } \\
\text { Type }\end{array}$ & Phy/WirelessPhyExt \\
\hline \hline MAC Type & Mac/802_11Ext \\
\hline \hline Interface Queue Type & Queue/DropTail/PriQueue \\
\hline \hline Link Layer Type & LL \\
\hline Antenna Model & Antenna/OmniAntenna \\
\hline Max Packet in Ifq & 50 \\
\hline \hline $\begin{array}{l}\text { Number of } \\
\text { Mobilenodes }\end{array}$ & 2 \\
\hline \hline Routing Protocol & AODV \\
\hline \hline Network X,Y & $10000 \mathrm{x} 10000$ \\
\hline \hline
\end{tabular}

\subsection{Steps to Run EvalVid on NS-2 on Ubuntu}

- Download video source file either CIF or QCIF format samples available in online which must be decoded into YUV sequence by using ffmpeg codec as shown figure 3.3

\section{\$ffmpeg -i a.264 a.y.uv}

- $\quad$ x264 a-I 30 -B 64 --fps 30 -o a1.264 --input-resa $352 \times 288$ a.yuv an raw video with 30 frames /sec will be created by $x 264$, where GOP length of 30 frames with no B frames as shown in figure 3.4. The CIF format can have a video resolution $352 \times 288$.

- MP4Box creates files of ISO mp4 containing the video samples .

MP4Box -hinta -mtu 1024 -fps 30 -add a1.264 a1.mp4

- $\quad$ ffmpeg -ia a1.mp4 a2.yuv

The above command line creates reference video to assess the video quality.

- $\quad$ The mp4trace tool is used to send a hinted mp4-file per RTP/UDP to a specified destination host The following command generates a video trace file ' $a$ ' is used for tranmission over wireless networks. This trace fie contains frame number, frame type (H,I,P and B frames), frame size, number of udp-packets, sender time.

./ mp4trace -f -s 192.168.0.2 12346 a1.mp4 > a

- Next step is to run the tcl script by the folloing command

\$ ns a2.tcl

- The video received by receiver get reconstructed. For this, the video and trace files are processed by etmp4

$$
\text { \$./etmp4a-f -0 s-a1 r-a1 a a1.mp4 a1 }
$$

- Decode .mp4 into .yuv format and then calculate the psnr

$$
\text { \$ ffmpeg -i a1.mp4 a2.yuv }
$$

\$./psnr 352288420 a.yuv a2.yuv > psnr.txt

\section{RESULTS AND ANALYSIS}

\subsection{Simulation Methodologies}

- Scenario 1: By varying the simulation time of video transmission for a particular speed of sink node. In this scenario, PDR, throughput and routing overhead calculated for each modulation and for adaptive modulation.

- Scenario 2: By varying the speed of the sink node for a complete video transmission. In this scenario, PDR, throughput, routing overhead,\% loss of frame/packet

\subsection{Performance Metrics}

- Packet Delivery Ratio (PDR) : It is the ratio of number of received packets and number of packets sent.

- Throughput : the packets received within given time.

- Routing Overhead : This metric is correlated with the number of route changes occurred in the simulation.

- $\%$ loss of frame/packet : It is a percentage of overall loss of I,P and B frames in a video. 
- Peak Signal Noise Ratio : It measures the difference between a reconstructed video file and the original video file

Table-5.1 Throughput for a complete Video transmission

\begin{tabular}{|l|l|l|l|l|}
\hline \multirow{2}{*}{\begin{tabular}{l} 
Speed of $\begin{array}{l}\text { Sink node } \\
\text { in m/s }\end{array}$ \\
\cline { 2 - 5 }
\end{tabular}} & $\begin{array}{l}\text { Throughput in Kbps } \\
\text { QAM }\end{array}$ & 16-QAM & QPSK & \\
\hline 10 & 0.0248 & 0.0511 & 0.0799 & 0.0939 \\
\hline 15 & 0.0169 & 0.0352 & 0.0537 & 0.0639 \\
\hline 20 & 0.0128 & 0.0261 & 0.0412 & 0.0492 \\
\hline 25 & 0.0104 & 0.0211 & 0.0336 & 0.04 \\
\hline 30 & 0.0088 & 0.0177 & 0.0277 & 0.0338 \\
\hline
\end{tabular}

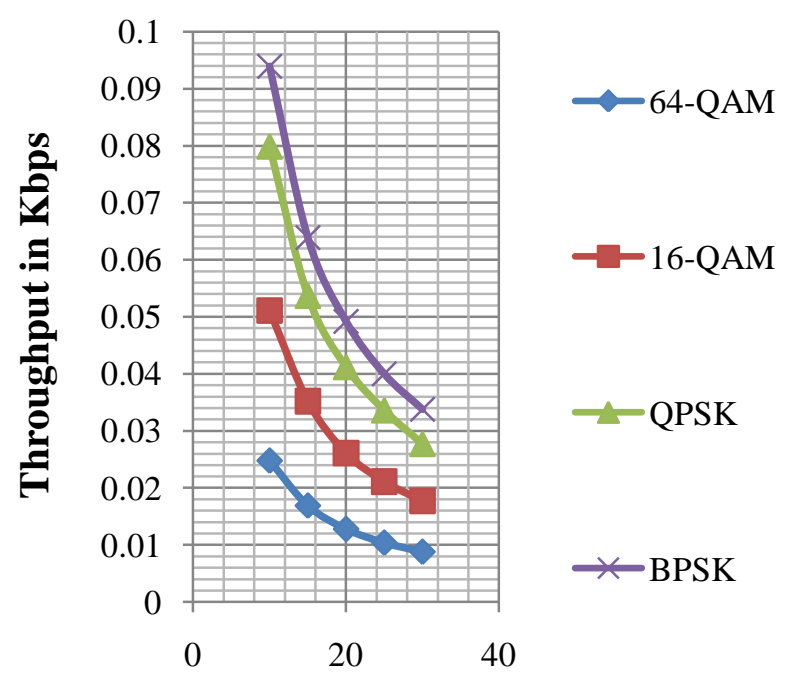

\section{Speed of sink node in $\mathrm{m} / \mathrm{s}$}

Fig 5.1 Throughput for a complete Video transmission

From the Table 6.1 and figure 6.1, observed that the lower order modulation yields better throughput compared to higher order modulation as the sink node speed increases. For each modulation throughput value decreases by increasing node speed.

Table -5.2 Throughput for Adaptive Modulation and Coding

\begin{tabular}{|l|l|}
\hline $\begin{array}{l}\text { Speed of Sink } \\
\text { node in } \mathbf{~ m} / \mathbf{s}\end{array}$ & $\begin{array}{l}\text { Throughput for Adaptive } \\
\text { Modulation and Coding }\end{array}$ \\
\hline 10 & 0.0939 \\
\hline 15 & 0.0639 \\
\hline 20 & 0.0492 \\
\hline 25 & 0.04 \\
\hline 30 & 0.0338 \\
\hline
\end{tabular}

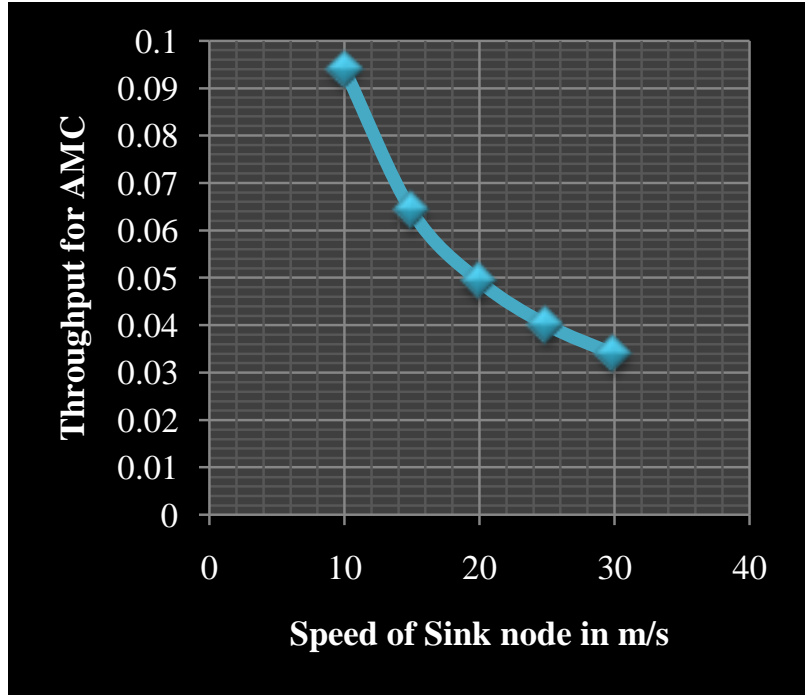

Fig 5.2 Throughput for Adaptive Modulation and Coding

From the Table 6.1 and figure 6.2, observed that throughput for AMC decreases as the sink node speed increases. But AMC yields better throughput compared to fixed modulation.

Similarly analysis can be done with different performance metrics like,PDR(packet delivery ratio), Routing Overhead, psnr etc to compare AMC with Different Modulation techniques

\section{CONCLUSION}

Modulation is the process of encoding information into signal for the purpose of transmission. It is performed at the physical layer. The performance of four modulations BPSK, QPSK, 16-QAM and 64-QAM are analyzed for video transmission over single-hop wireless networks by considering the sink node movement. The performance metrics like PDR, throughput, routing overhead, PSNR and frame/packet loss have been taken for each modulation. Evalvid tool is used in NS2 for video transmission over wireless networks. This tool calculates the frame/packet loss and PSNR metrics. From the simulation, observed that the lower order modulation yields better results compared to higher order modulation.

AMC is a method of changing modulation scheme based on wireless link condition to increase the spectral efficiency. The four modulations are adapted based on packet drooping time of each modulation for a particular speed of sink node. From the simulation, observed that AMC have better results in terms of throughput, PDR, frame/packet loss and PSNR compared to fixed modulation.

In future work, the performance of AMC over multi-hop networks for video transmission can be analyzed. Furthermore the performance of AMC over wireless networks with different types of routing protocols can be compared because in this project only routing protocol AODV is used. 


\section{REFERENCES}

[1] Dalei Wu, Cross-Layer Optimized Wireless Multimedia Networking,A Dissertation Presented to the Faculty of The Graduate College at the University of Nebraska in Partial Fulfilment of Requirements For the Degree of Doctor of Philosophy, November 2010.

[2] Q. Liu, S. Zhou, and G. B. Giannakis, Cross-layer Combining of Adaptive Modulation and Coding with Truncated ARQ over Wireless Links, IEEE Transactions on Wireless Communications, vol 3, pp 1746-1755, September 2004.

[3] Q. Liu, S. Zhou, and G. B. Giannakis, Queuing with Adaptive Modulation and Coding over Wireless Links: Cross-Layer Analysis and Design,IEEE Transactions on Wireless Communications, vol 4, no.3, pp 11421153, May 2005.

[4] Xin Wang, Qingwen Liu and Georgios B. Giannakis, Analyzing and Optimizing Adaptive Modulation Coding Jointly With ARQ for QoS-Guaranteed Traffic,IEEE Transactions on Vehicular Technology,vol 56, no. 2, March 2007.

[5] Fantacci, Marabissi, Tarchi and Habib, Adaptive modulation and coding techniques for OFDMA systems, IEEE Transactions on Wireless Communications, Vol 8 , no.9,pp 4876 - 4883, September 2009.

[6] FeiPeng, Arizona Univ, Tucson, AZ, Jinyun Zhang, Ryan and W.E,Adaptive Modulation and Coding for IEEE 802.11n, IEEE Transactions on Wireless Communications and Networking Conference, 2007.

[7] C. Perkins, E. Belding-Royer, S. Das,Ad hoc OnDemand Distance Vector (AODV) Routing,Network Working Group, published in RFC 3561, July 2003.

[8] Theodore and S. Rappaport, Wireless Communications Principles and Practice, 2nd Edition, Pearson Education Asia, 2002.

[9] Modulation theory detail explanation, http://www.radio-electronics.com.

[10] Memon T.D., Ghangro W., Chowdhry B.S., and Shaikh A.A., Quadrature Phase Shift Keying modulator \& demodulator for Wireless Modem, IEEE transaction on Computer, Control and Communication, Feb 2009.

[11] Svensson A., An Introduction to Adaptive QAM Modulation Schemes for Known and Predicted Channels, In Proceedings of the IEEE, Vol 95, no. 12,December 2007.

[12] C. H. Ke, C. K. Shieh, W. S. Hwang, A. Ziviani, An Evaluation Framework for More Realistic Simulations of MPEG Video Transmission, Journal of Information Science and Engineering, vol. 24, no. 2, pp.425-440, March 2008 (SCI)

[13] H. S,chulzrinne, S. Casner, and R. Frederick, RTP: A Transport Protocol for Real-Time Applications, Network Working Group of the Internet Engineering Task Force (IETF) and first published in 1996 as RFC 1889, superseded by RFC 3550 in 2003.

[14] C. H. Ke, C. K. Shieh, W. S. Hwang, and A. Ziviani, Improving video transmission on the Internet, IEEE Potentials Magazine, vol 26, no 1, pp 16-19, 2007
[15] Network simulator(NS-2) , http://www.isi.edu /nsnam/ns

[16] Evalvid document, http://www2.tkn.tuberlin.de/research/evalvid/EvalVid/docevalvid.html

[17] Usage of evalvid, http://www2.tkn.tuberlin.de/research/evalvid/fw.html

[18] Usage of evalvid on ns2, http://totalgeekout.blogspot.in/2013/04/evalvid-on-ns2-on-ubuntu-1204.html

[19] Installation of evalvid on ns2, http://csie.nqu.edu.tw/smallko/ns2_old/Evalvid_in_NS 2.htm

[20] To solve the error while running etmp4, https://airtoncs. wordpress.com/2013/06/29/bug-fixingon-etmp4-tool 